\title{
High efficiency of graded index photonic crystal as an input coupler
}

Atilla Ozgur Cakmak, Evrim Colak, Humeyra Caglayan, Hamza Kurt, and Ekmel Ozbay

Citation: Journal of Applied Physics 105, 103708 (2009);

View online: https://doi.org/10.1063/1.3130403

View Table of Contents: http://aip.scitation.org/toc/jap/105/10

Published by the American Institute of Physics

\section{Articles you may be interested in}

The focusing effect of graded index photonic crystals

Applied Physics Letters 93, 171108 (2008); 10.1063/1.3009965

Self-focusing media using graded photonic crystals: Focusing, Fourier transforming and imaging, directive emission, and directional cloaking

Journal of Applied Physics 110, 053103 (2011); 10.1063/1.3630116

Focusing of light beyond the diffraction limit by randomly distributed graded index photonic medium Journal of Applied Physics 120, 243102 (2016); 10.1063/1.4972980

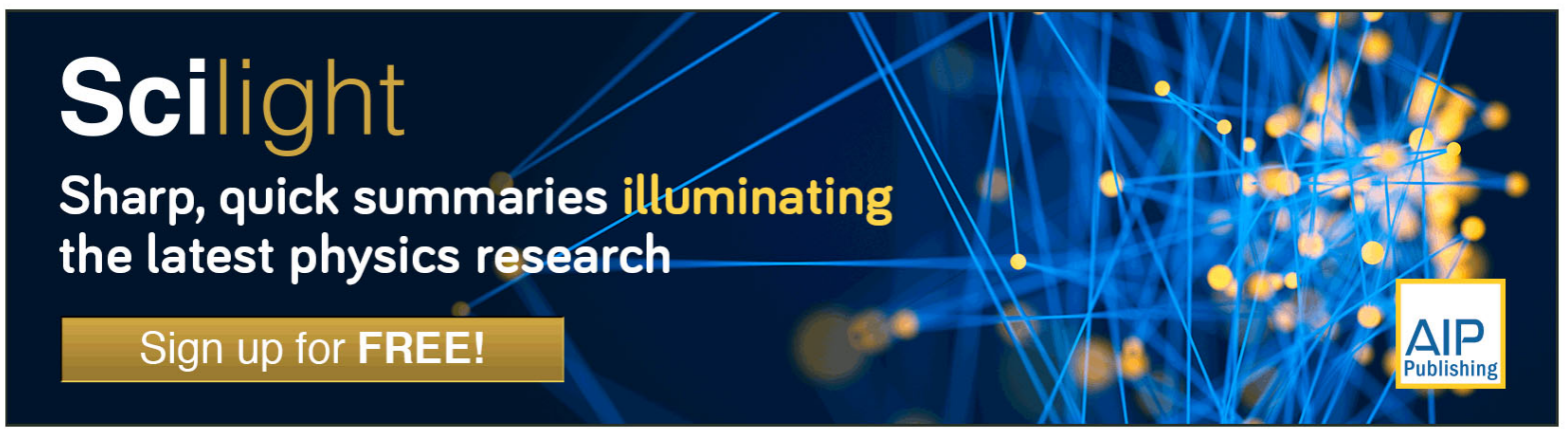




\title{
High efficiency of graded index photonic crystal as an input coupler
}

\author{
Atilla Ozgur Cakmak, ${ }^{1, a)}$ Evrim Colak, ${ }^{1}$ Humeyra Caglayan, ${ }^{1}$ Hamza Kurt, ${ }^{2}$ and \\ Ekmel Ozbay ${ }^{1}$ \\ ${ }^{1}$ Department of Physics, Nanotechnology Research Center and Department of Electrical and Electronics \\ Engineering, Bilkent University, 06800 Ankara, Turkey \\ ${ }^{2}$ Department of Electrical and Electronics Engineering, TOBB University of Economics and Technology, \\ 06560 Ankara, Turkey
}

(Received 17 February 2009; accepted 5 April 2009; published online 26 May 2009)

\begin{abstract}
A graded index photonic crystal (GRIN PC) configuration was placed at the input side of a photonic crystal waveguide $(\mathrm{PCW})$ in order to efficiently couple the light waves into the waveguide. We compared the transmission efficiencies of light in the absence and presence of the GRIN PC structure. We report a significant improvement in coupling when the GRIN PC is incorporated with the PCW. The intensity profiles were obtained by carrying out the experiments at microwave frequencies. Finite difference time domain based simulations were found to be in good agreement with our experimental results. (C) 2009 American Institute of Physics. [DOI: 10.1063/1.3130403]
\end{abstract}

\section{INTRODUCTION}

The developments of science in recent years have allowed photonic crystals (PCs) to take their place among various applicable research areas rather than just being mentioned as an obscure physics topic. ${ }^{1,2}$ The periodic arrangements of the PC structure offer superior performance over their conventional dielectric counterparts in optics. As a consequence, PC based devices have come to be fully appreciated due to their key features in controlling the flow of electromagnetic (EM) waves. A photonic crystal waveguide (PCW) is an excellent example that has long been both theoretically and experimentally investigated. ${ }^{3-5}$ PCWs are created by introducing line defects. These line defects guide the light with considerably reduced losses over sharp bends by strongly confining the propagating modes with the help of the Bragg reflection mechanisms. Thus, their wide usage in the field brought up the challenge of efficiently coupling light into PCWs. The mismatch between the modes of the external lightwave circuits and the PCW was accepted as the main reason for the poor coupling figures. Hence, several ways of tackling this problem were proposed. Adiabatically tapered fibers and dielectric waveguides were suggested as a solution to this obstacle. ${ }^{6,7}$ The employment of gratings ${ }^{8}$ and $\mathrm{J}$-couplers, founded on the principles of parabolic mirrors, were adapted to overcome the difficulties. ${ }^{9,10}$ Yet, the main attraction was directed toward the utilization of the tapered PCWs that facilitate adiabatic mode conversion. ${ }^{11-20}$ The supporting theoretical studies show promise for considerably high coupling efficiencies when we make use of these tapered PCWs. ${ }^{21,22}$ Nonetheless, tapered PCWs have simultaneously led to serious drawbacks. Many of the approaches have depended on the complicated manufacturing steps. The slow reshaping process of the incident beam has required relatively long periods of the PCW to be sacrificed at both ends.

Efforts have been initiated to search for alternative meth-

\footnotetext{
a) Author to whom correspondence should be addressed. Electronic mail: atilla@ee.bilkent.edu.tr.
}

ods that can compete and even replace the existing schemes. In that respect, the self collimation abilities of the PCs has received much attention. ${ }^{23-26}$ The graded index (GRIN) version of the $\mathrm{PC}$ is a distinguished candidate in the literature for realizing the self-focusing phenomena. A theoretical work was devoted to understand the critical design stages of the GRIN PCs. ${ }^{27}$ Following that article, the GRIN PCs were integrated with PCWs to yield high coupling factors. ${ }^{28} \mathrm{~A}$ GRIN PC that was composed of air holes was discussed in Ref. 28, in which the index variation was achieved by properly adjusting the radius of the holes. Numerical analysis was carried out in order to emphasize the enhanced coupling figures. However, an experimental demonstration was not put forward. Therefore, the main objective of this study has been to experimentally verify the improved coupling that was attained with the assistance of the GRIN PC in the microwave regime. In the remaining part of the present paper, the experimental and simulation results are provided. Even though the finalized design of the GRIN structure and its theoretical examinations were the main scope of a recently published work, ${ }^{29}$ a quick overview of the functionalized GRIN PC and the PCW is presented first. The experiments were accompanied by the numerical outcomes. Finally, the concluding remarks are laid out together with the performance issues concerning the coupling efficiency.

\section{PCW}

The scalability of the Maxwell's equations enables us to make analogies between the optical and microwave domains. An accurately scaled PCW, operating at the microwave frequencies, is a 2D representation of its optical equivalent. Figure 1(a) depicts the top view of the PCW that comprises sufficiently long (much longer than the operational wavelength) alumina rods with a dielectric constant of $\varepsilon=9.61$. The PCW is on the $x-z$ plane and has a lattice constant of $a=7 \mathrm{~mm}$. The square lattice PCW stretches out 11 and 29 periods along the $x$ and $z$ directions, respectively. A row of rods starting from the 15 th rod on the $z$-axis was removed to create the line defect. Figure 1(b) illustrates the dispersion 
(a)

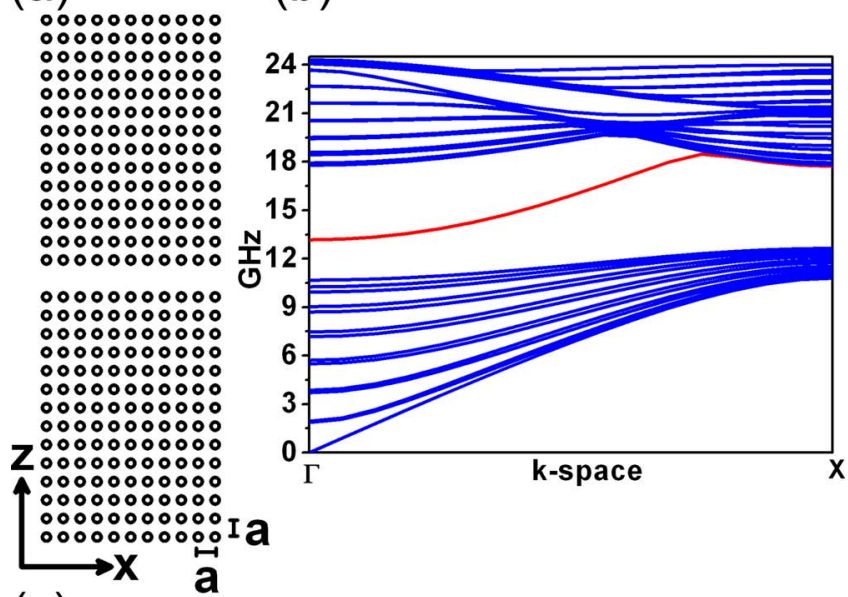

(c)

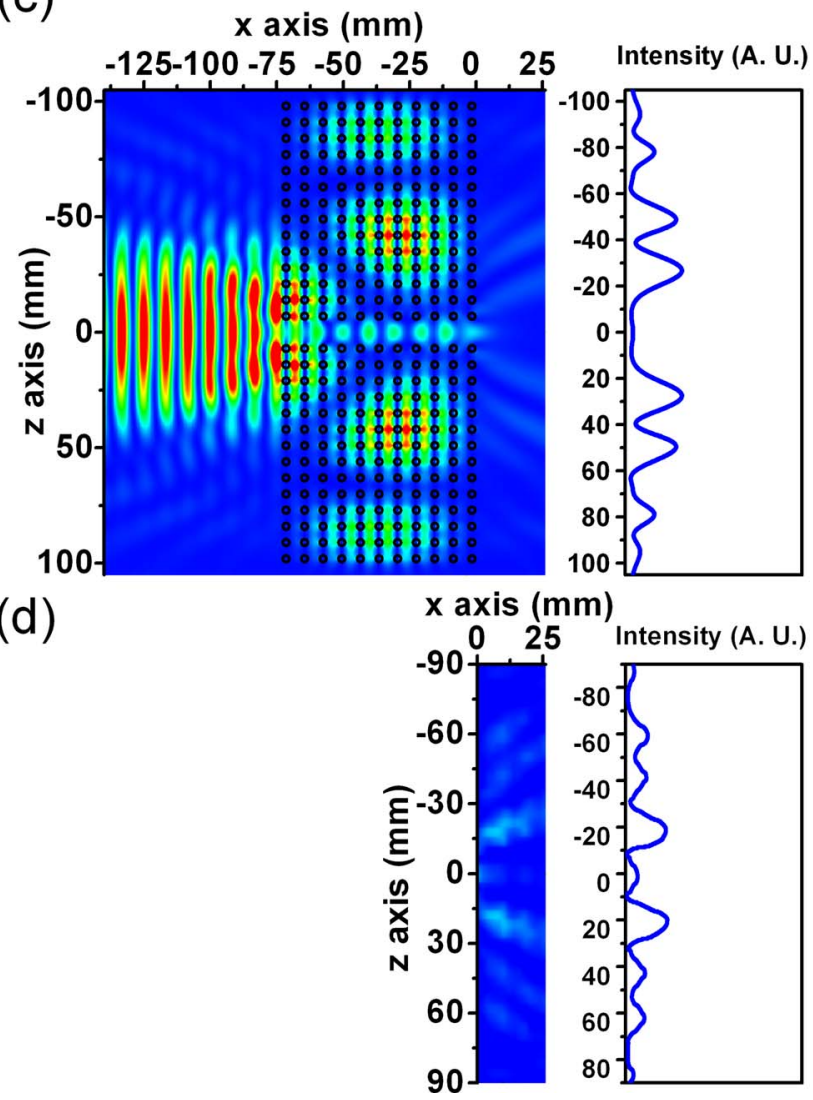

FIG. 1. (Color online) (a) Top view of the PCW structure. Alumina rods with $\varepsilon=9.61$, standing in the air $(n=1)$, lattice constant $a=7 \mathrm{~mm}$. (b) Dispersion diagram of the PCW structure along the $\Gamma-X$ direction for TM polarization. The defect band is illustrated with the red line. (c) Simulation and (d) experimental results of the intensity distributions of the electric field $\left(E_{y}\right)$ at $18 \mathrm{GHz}$. A slice of the intensity distribution at the output side is also given at the right hand side of the main figure.

graph of the PCW for the TM polarization $\left(E_{y}\right.$ parallel to the rods) in the $\Gamma-X$ direction. The defect band is highlighted with a red line while the rest of the blue bands all stay outside the band gap. The defect band supports a wide range of modes, including $18 \mathrm{GHz}$, which is designated as our working frequency and is restricted by the GRIN PC. The restriction is that the PCW must sustain the propagating mode while the GRIN PC allows high transmission. Simulations have been performed using a commercial finite difference time domain (FDTD) tool called RSOFT. Figure 1(c) shows the intensity distribution of the electric field. A single frequency $(18 \mathrm{GHz})$, wide Gaussian beam with a full width at half maximum (FWHM) of $3 \lambda$ ( $\lambda$ being the operational wavelength) was launched from a distance toward the PCW. Only a small portion of the input beam was observed to be coupled to the waveguide due to the mode mismatches. This is analogous to what is happening at the optical frequencies as in the case of the butt coupling of different waveguide widths. A dielectric waveguide mode often also suffers from high coupling losses at the PCW interfaces. Moreover, as it can also be pointed out in Fig. 1(c), a portion of the incident beam was not localized within the line defect, since $18 \mathrm{GHz}$ is close to the air band [Fig. 1(b)]. Thus, the portion of the incident light hitting the PCW side walls in turn leaks out through the structure. Consequently, the wave cannot be said to be confined to the waveguide, but rather tends to spread out. The adjacent figure corresponds to the intensity profile at the exit side of the PCW. The slice was taken at a distance of $5 \mathrm{~mm}$ (all of the distances are in millimeters). The combination of the diffraction mechanisms and the weak confinement of the wave hinder the overall performance and hence do not permit the high transmission. The next step was the realization of the experimental setup. A conventional horn antenna with the same FWHM value was utilized to send the incident beam to the PCW from $70 \mathrm{~mm}$ away. A monopole antenna was used to collect the beams at the output side while the network analyzer kept record of the measured intensities. The shortcomings of our experimental setup compelled us to scan only the output section of the PCW. Figure 1(d) is the measured intensity distribution at the exit side of the PCW. The intensity profile suggests that the diffraction mechanisms and the coupling losses have once again governed the transmission experiment, which is consistent with the numerical scenario.

\section{GRIN PC}

The GRIN PC structure is designed by properly arranging the shifts in the longitudinal direction while keeping the lattice spacing constant along the $x$-axis. It is portrayed in Fig. 2(a). The variation is sustained such that the density of the rods is denser at the center of the GRIN PC. The EM wave prefers to travel at higher refractive index regions and as a result, a self-focusing phenomenon is conveniently observed. The GRIN PC has translational symmetry over the $x$-axis. The symmetry axis divides the GRIN PC in such a way that it has 14 rods in both of the divided segments. The separation between two rods is determined by two variables, the constant factor of $b_{0}=0.5 a$ and $\Delta b=0.15 a$, which is altered at every two lattices. A seven layered GRIN PC is considered for our particular case, but it was already shown that even a four layered GRIN PC would be enough to exhibit comparable self-focusing effects. ${ }^{29}$ First, the simulations were run to acquire the intensity distributions of the electric field when the GRIN PC was excited with a wide Gaussian beam. The numerical results predict that the shape of the wavefronts of the incoming wave is modified once the beam enters the GRIN PC. The accompanying intensity pro- 
(a)

(b)
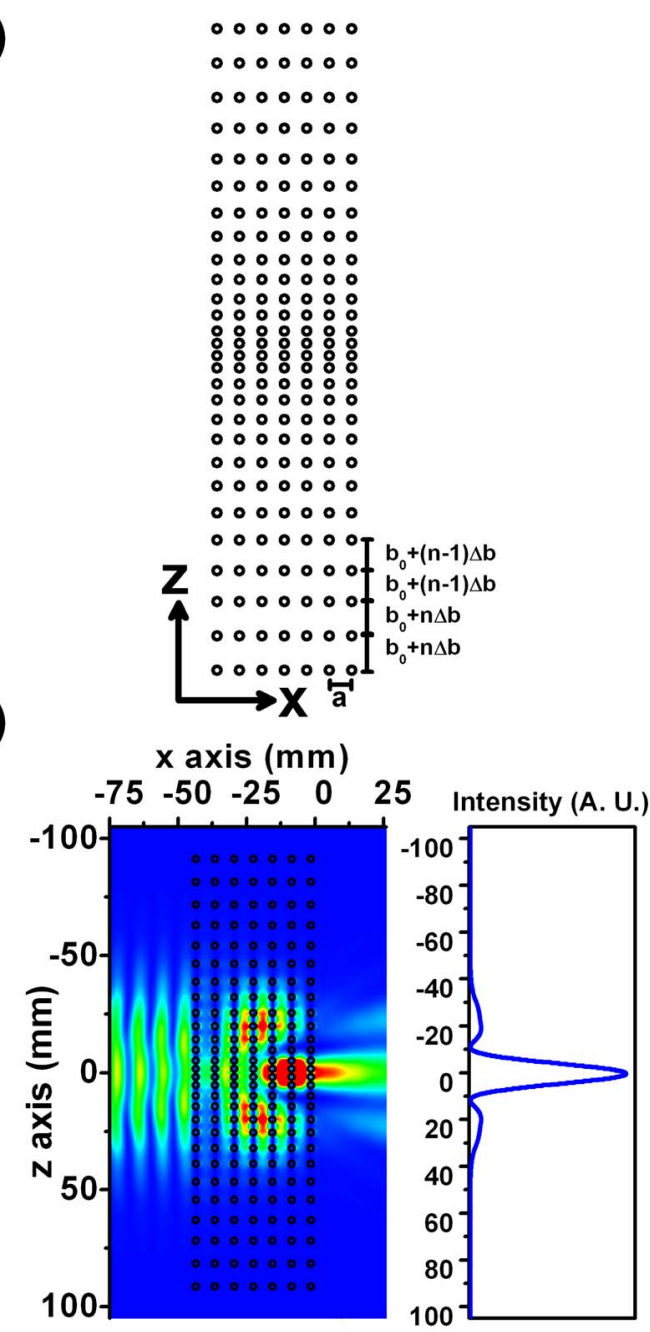

(c)

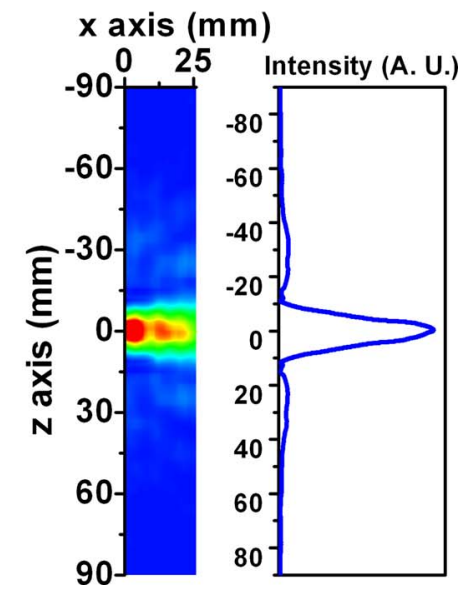

FIG. 2. (Color online) (a) Top view of the GRIN PC structure, composed of alumina rods, $b_{0}=0.5 a$ and $\Delta b=0.15 a$. (b) Simulation and, (c) experimental results of the intensity distributions of the electric field $\left(E_{y}\right)$ at $18 \mathrm{GHz}$. A slice of the intensity distribution at the output side is also given at the right hand side of the main figure.

file in Fig. 2(b) displays that a narrowed beam was generated as the product. A similar experimental setup was used to scan the output side of the GRIN PC. The experimental results reveal a very good agreement with the numerical conclusions. The measurements are shown in Fig. 2(c), and they imply that the GRIN PC acts like a lens with a certain focal (a)

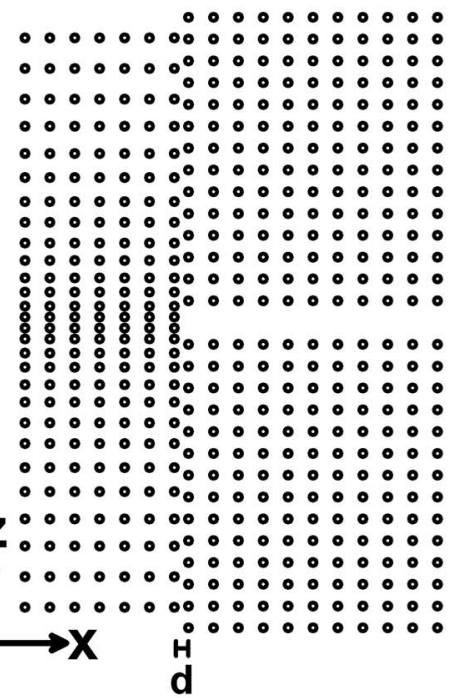

(b)

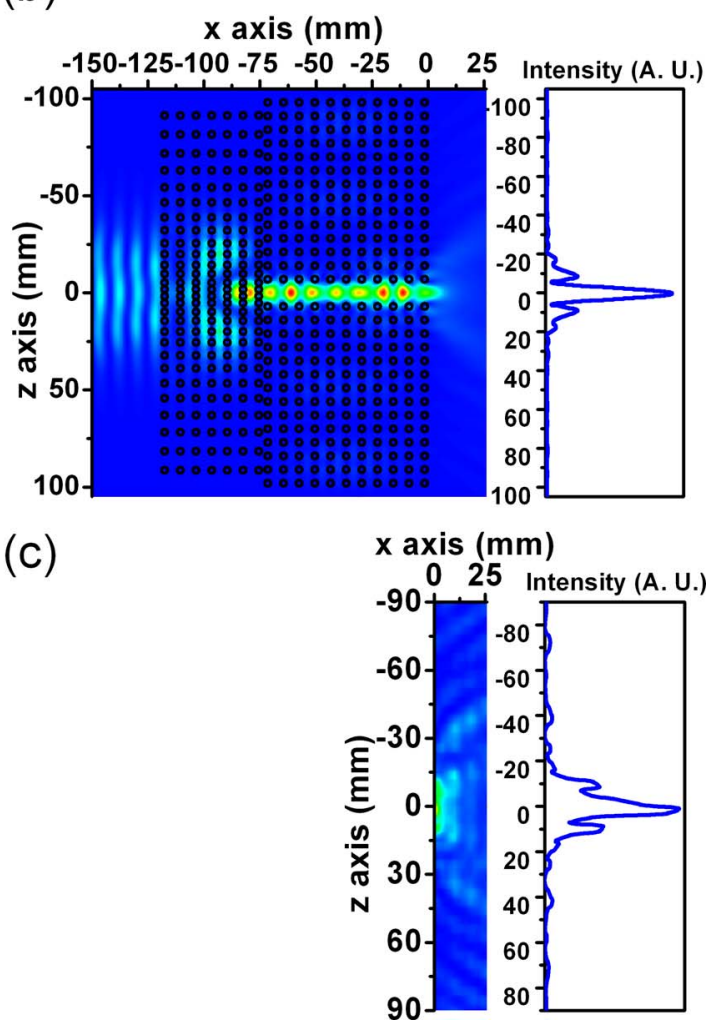

FIG. 3. (Color online) (a) Top view of the overall structure $d=4 \mathrm{~mm}$. (b) Simulation and (c) experimental results of the intensity distributions of the electric field $\left(E_{y}\right)$ at $18 \mathrm{GHz}$. A slice of the intensity distribution at the output side is also given at the right hand side of the main figure.

length. The focal point is approximately found to be $4 \mathrm{~mm}$ away from the surface of the GRIN PC.

\section{GRIN PC+PCW}

In the next stage, the GRIN PC is cooperated along with the PCW to increase the coupling efficiency. The wide beam was to be squeezed down prior to being fed to the PCW by taking advantage of the focusing effect of the GRIN PC. It was experimentally checked to ensure that the optimum lateral spacing $d$ between the two configurations was $4 \mathrm{~mm}$. Then, the PCW was positioned around the focal point of the 
(a)

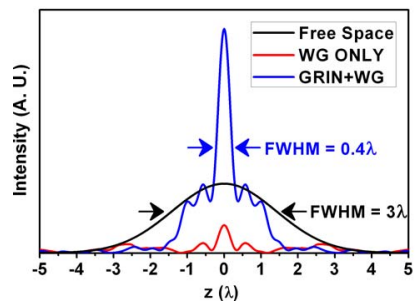

(b)

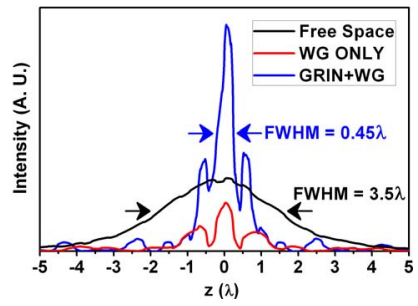

FIG. 4. (Color online) The intensity profiles at the output surface of the PCW: GRIN+PCW (solid blue line) and PCW ONLY (solid red line). The free space intensity profile (solid black line) has also been given as a reference. (a) Numerical and (b) experimental results.

GRIN PC to give rise to the highest transmission figures, as shown in Fig. 3(a). The FDTD results of Fig. 3(b) assure enhanced transmission figures and immensely reduced coupling losses. Regardless of the weak confinement of the PCW at $18 \mathrm{GHz}$, the spatially narrowed EM wave, due to the GRIN PC, propagates without significant broadening and reaches the exit side while keeping its form. The numerical results were once again confirmed by the experimental measurements. When the fields were probed at the exit interface of the PCW, it was observed that the profiles resembling the numerical outcomes were obtained experimentally, as in Fig. 3(c). It can be identified that this narrow mode does not have the capability to cover long distances after having departed the PCW. The diffractions are likely to destroy the shape of the outgoing wave. An output coupler might be needed to extract the waves from the PCW, but for our own purposes, we are contented with the validation of the improved coupling at the input side for the time being.

When the intensity profiles were collected only at the exit surface of the PCW, we witnessed the situations that are given in Fig. 4. Figure 4(a) was drawn up solely based on the FDTD calculations, whereas Fig. 4(b) shows the experimental results. Both of the figures impressively demonstrate the effectiveness of using the GRIN PC as the input coupler. A wide incident beam with a FWHM of $3 \lambda$ underwent a mode conversion ratio of 7.5 and arrived at the other side with a FWHM of $0.4 \lambda$. Similarly, the measurements offered a mode conversion ratio of 7.7. Given that the FWHM values are comparably smaller than the total window size of $10 \lambda$, integrals of the intensity profiles at the exit side should provide an estimation of the coupling efficiency for the GRIN PC. The Simpson's numerical integration technique was adapted for a range of $-5 \lambda \leq z \leq 5 \lambda$. Table I summarizes the coupling

TABLE I. The calculated numerical and experimental coupling efficiencies of the system with the inclusion of the GRIN PC structure

\begin{tabular}{lcc}
\hline \hline & $\begin{array}{c}\text { Simulation } \\
(\mathrm{dB})\end{array}$ & $\begin{array}{c}\text { Experiment } \\
(\mathrm{dB})\end{array}$ \\
\hline $\int_{-5 \lambda}^{5 \lambda}\left|E_{y}\right|_{\mathrm{GRIN}+\mathrm{PCW}}^{2}$ & & \\
$\int_{-5 \lambda}^{5 \lambda}\left|E_{y}\right|_{\mathrm{PCW}}^{2}$ & 6.35 & 5 \\
\hline \hline
\end{tabular}

efficiencies of the simulations and experiments. The inclusion of the GRIN PC leads to 6.35 and $5 \mathrm{~dB}$ improvements in coupling mechanism numerically and experimentally, respectively.

\section{CONCLUSION}

In summary, the presented study is a proof of the utilization of the GRIN PC as an efficient input coupler for the PCW. As a result, the GRIN PC has been experimentally shown to yield a coupling efficiency of $5 \mathrm{~dB}$ over the single PCW at $18 \mathrm{GHz}$. The successful experimental outcomes were backed up with detailed numerical analysis. Throughout the paper, we attempted to make analogies between the microwave and optical domains as much as possible in order to address the complementary problem at the optical frequencies. PC manufacturing techniques have been well founded in recent years. We believe that it is manageable to bring both the GRIN PC and the PCW to the optical domain. A horn antenna can be thought of as a representative of the dielectric waveguides. Our proposed method can then be applied to attack the input coupling losses between PC structures and other lightwave circuits.

\section{ACKNOWLEDGMENTS}

This work is supported by the European Union under the projects EU-PHOME, and EU-ECONAM, and TUBITAK under Project Nos. 105A005, 106E198, and 107A004. One of the authors, E.O., also acknowledges partial support from the Turkish Academy of Sciences.

${ }^{1}$ C. M. Soukoulis, Nanotechnology 13, 420 (2002).

${ }^{2}$ P. Russell, Science 299, 358 (2003).

${ }^{3}$ R. D. Meade, A. Devenyi, J. D. Joannopoulos, O. L. Alerhand, D. A. Smith, and K. Kash, J. Appl. Phys. 75, 4753 (1994).

${ }^{4}$ B. Temelkuran and E. Ozbay, Appl. Phys. Lett. 74, 486 (1999).

${ }^{5}$ G. Manzacca, D. Paciotti, A. Marchese, M. S. Moreolo, and G. Cincotti, Photonics Nanostruct. Fundam. Appl. 5, 164 (2007).

${ }^{6}$ Y. Xu, R. K. Lee, and A. Yariv, Opt. Lett. 25, 1756 (2000).

${ }^{7}$ J. Bauer and S. John, Phys. Rev. A 77, 013819 (2008).

${ }^{8}$ D. Taillaert, W. Bogaerts, P. Bienstman, T. F. Krauss, P. V. Daele, I. Moerman, S. Verstuyft, K. D. Mesel, and R. Baets, IEEE J. Quantum Electron. 38, 949 (2002).

${ }^{9}$ D. W. Prather, J. Murakowski, S. Shi, S. Venkataraman, A. Sharkawy, C. Chen, and D. Pustai, Opt. Lett. 27, 1601 (2002).

${ }^{10}$ T. Dillon, J. Murakowski, S. Shi, and D. Prather, Opt. Lett. 33, 896 (2008).

${ }^{11}$ C. W. Chang, S. C. Cheng, and W. F. Hsieh, Opt. Commun. 242, 517 (2004).

${ }^{12}$ A. A. Green, E. Istrate, and E. H. Sargent, Opt. Express 13, 19 (2005).

${ }^{13}$ P. Sanchis, J. Garcia, J. Marti, W. Bogaerts, P. Dumon, D. Taillaert, R. Baets, V. Wiaux, J. Wouters, and S. Beckx, IEEE Photon. Technol. Lett. 16, 2272 (2004).

${ }^{14}$ A. Mekis and J. D. Joannopoulos, J. Lightwave Technol. 19, 861 (2001).

${ }^{15}$ A. Hakansson, P. Sanchis, J. S. Dehesa, and J. Marti, J. Lightwave Technol. 23, 3881 (2005).

${ }^{16}$ P. Bienstman, S. Assefa, S. G. Johnson, J. D. Joannopoulos, G. S. Petrich, and L. A. Kolodziejski, J. Opt. Soc. Am. B 20, 1817 (2003).

${ }^{17}$ E. H. Khoo, A. Q. Liu, and J. H. Wu, Opt. Express 13, 7748 (2005).

${ }^{18}$ A. Talneau, P. Lalanne, M. Agio, and C. M. Soukoulis, Opt. Lett. 27, 1522 (2002).

${ }^{19}$ P. Pottier, M. Gnan, and R. M. De La Rue, Opt. Express 15, 6569 (2007).

${ }^{20}$ J. P. Hugonin, P. Lalanne, T. P. White, and T. F. Krauss, Opt. Lett. 32, 2638 (2007).

${ }^{21}$ S. G. Johnson, P. Bienstman, M. A. Skorobogatiy, M. Ibanescu, E. Lidorikis, and J. D. Joannopoulos, Phys. Rev. E 66, 066608 (2002).

${ }^{22}$ M. Qiu, K. Azizi, A. Karlsson, M. Swillo, and B. Jaskorzynska, Phys. Rev. 
B 64, 155113 (2001)

${ }^{23}$ D. W. Prather, S. Shi, J. Murakowski, G. J. Schneider, A. Sharkawy, C. Chen, B. L. Miao, and R. Martin, J. Phys. D 40, 2635 (2007).

${ }^{24}$ H. Kosaka, T. Kawashima, A. Tomita, M. Notomi, T. Tamamura, T. Sato, and S. Kawakami, Appl. Phys. Lett. 74, 1212 (1999).

${ }^{25}$ J. Witzens, M. Loncar, and A. Scherer IEEE J. Sel. Top. Quantum Elec- tron. 8, 1246 (2002).

${ }^{26}$ L. Wu, M. Mazilu, and T. F. Krauss, J. Lightwave Technol. 21, 561 (2003). ${ }^{27}$ H. Kurt and D. S. Citrin, Opt. Express 15, 1240 (2007).

${ }^{28}$ H. Kurt and D. S. Citrin, IEEE Photon. Technol. Lett. 19, 1532 (2007).

${ }^{29}$ H. Kurt, E. Colak, O. Cakmak, H. Caglayan, and E. Ozbay, Appl. Phys. Lett. 93, 171108 (2008). 